\title{
Mild-to-severe traumatic brain injury in children: altered cytokines reflect severity
}

\author{
Emer Ryan 1,2,3,4 ${ }^{0}$, Lynne Kelly ${ }^{1,2,3}$, Catherine Stacey ${ }^{1,2,3}$, Dean Huggard ${ }^{1,2,3,10}$, Eimear Duff ${ }^{1,2,3}$, \\ Danielle McCollum 1,2,3,4,9, Ann Leonard ${ }^{5}$, Gerard Boran ${ }^{5}$, Dermot R. Doherty ${ }^{6}$, Turlough Bolger ${ }^{1,4}$ and \\ Eleanor J. Molloy $1,2,3,4,7,8^{*}$
}

\begin{abstract}
Background: Paediatric traumatic brain injury (TBI) is recognised to have significant longer-term neurocognitive effects. Childhood is a time of high risk for head injury. Functional recovery is variable with a combination of any or all of physical, cognitive and emotional impairment. Immune activation and alteration in cytokine levels are present following TBI which may differ from adults.
\end{abstract}

Methods: Pro- and anti-inflammatory cytokines including Interleukin (IL)-2, IL-4, IL-6, IL-8, IL-10, IL-17A, Tumor Necrosis Factor (TNF)-a and Interferon (IFN)- $\gamma$ were examined at baseline and following in vitro treatment with endotoxin of whole blood, in the following children: severe TBI (sTBI: initial Glasgow coma scale(GCS) $\leq 8$ ), mild TBI (mTBI; GCS 14/15) at 0-4d and at 10-14d post-TBI and compared to healthy age-matched controls.

Results: The study enrolled 208 children, including 110 with TBI cohort ( $n=104$ mild; 6 severe) and controls $(n=98)$. At baseline all children with TBI had increased IL-6. The mTBI group had significantly increased IFN- $\gamma$ versus controls. In STBI at baseline, IFN- $\gamma$ was decreased compared to controls. At baseline IL-8, IL-10, IL-17A, and TNF-a were decreased in mTBI compared to controls. This persisted at 2 week post-mTBI. The AUC for detecting mTBI was $0.801 \mathrm{Cl}(0.73-086)$ using IL6/IL10 ratio. mTBI showed a greater fold change in IL-8 and TNF-a in response to endotoxin stimulation, a response that persisted at 2 weeks. Children with sTBI did not have a significant IL-6 response to endotoxin, but did show an increase in IL-17A.

Conclusion: Children with all TBI including mTBI show altered cytokine profiles and altered endotoxin responses. Although cytokines increased in sTBI especially in response to endotoxin, suppressed responses were found in mTBI coupled with persistent immune dysfunction post-injury.

Keywords: Traumatic Brain injury, Concussion, Inflammation, Cytokines, Innate immunity

\section{Background}

Traumatic brain injury is a global cause of preventable death and disability and a common presentation to the emergency department. Each year 3\% of all children under 5 years will present to the emergency department with head injury [1]. In England and Wales, of 1.4 million

\footnotetext{
*Correspondence: eleanor.molloy@tcd.ie

${ }^{1}$ Department of Paediatrics, Trinity College, The University of Dublin, Dublin, Ireland
}

Full list of author information is available at the end of the article emergency department visits a year for head injuries, half are under 15 years. Mild traumatic brain injury (mTBI) accounts for the majority (90\%) of traumatic brain injury [2]. The paediatric brain responds differently to injury for a variety of physical and developmental reasons, as well as the maturity of the immune system [3]. Inflammatory responses to injury changes across the lifespan. Exploring the age-related inflammatory component of TBI is important for our understanding and therapeutic interventions [4]. The consequences of more severe TBI original author(s) and the source, provide a link to the Creative Commons licence, and indicate if changes were made. The images or other third party material in this article are included in the article's Creative Commons licence, unless indicated otherwise in a credit line to the material. If material is not included in the article's Creative Commons licence and your intended use is not permitted by statutory regulation or exceeds the permitted use, you will need to obtain permission directly from the copyright holder. To view a copy of this licence, visit http://creativecommons.org/licenses/by/4.0/. The Creative Commons Public Domain Dedication waiver (http://creativeco mmons.org/publicdomain/zero/1.0/) applies to the data made available in this article, unless otherwise stated in a credit line to the data. 
in children are catastrophic, particularly, before neuronal networks are laid down. The consequence of mTBI in children, longer term is less well-established. Despite this, there are no therapeutic agents.

Neuronal injury results in a cascade of neuroinflammation with activation of glia, the release of inflammatory mediators and the recruitment of systemic immune cells. The composition of immune cells in the peripheral blood changes as children mature, with a lymphocyte increase from 6 months to adult and a decrease in neutrophils in teenage years [5]. The immune response to infective and inflammatory stimuli changes over a lifetime. An experimental animal model shows that TBI induces widespread innate and adaptive immune changes in myeloid progenitors, thymus and $\mathrm{T}$ cells months after injury [6]. Following brain injury in the neonatal period, by school age, children had chronically altered cytokine profiles with elevated serum IL-2, IL-6 and IL-8 [7]. Deficiency or overexpression of IL-6 impair the recovery phase of TBI. In the acute stage of severe adult traumatic brain injury (sTBI), IL-6 and IL-8 show an early increase [8]. Cerebrospinal fluid showed high levels of IL- 6 and IL-10 in earlier sTBI studies in children [9]. Serum IL-6, IL-8 and IL-10 predict poorer neurological outcomes in paediatric sTBI [10] Increased serum IL-6 in mTBI was significantly associated with the duration of symptoms in post concussive syndrome for mTBI in high school and college football players [11]. Early immune changes in the wake of TBI plays a role in the high rate of nosocomial infection in children [12].

Both IFN- $\gamma$ and IL- 4 have been studied in the context of TBI, but no study has generated conclusive evidence in their roles in TBI [13]. Interleukin-10 is known for its anti-inflammatory properties. In IL-10 knockout mice brain injury lesions are larger than wild-type mice [14] and increased IL-10 levels also correlate with hospital mortality in adults following sTBI [15]. A major role in inflammation is also played by TNF- $\alpha[16,17]$. In a rat model, the TNF- $\alpha$ antagonist Etanercept, administered early after TBI, reduced the secondary insults from oxidative stress and apoptosis with less neuronal loss [18]. The adaptive immune system, however, is less welldescribed in TBI. Interleukin-17A produced mainly by specialised T cells, Th17 and $\gamma \delta \mathrm{T}$ cells, plays a role in acute brain ischaemia, stroke [19] and multiple sclerosis [20]. It is also implicated in brain-gut signalling [21].

Here we describe the cytokine response in children with TBI compared to controls. As TBI has immunomodulating effects, we aimed to examine the cytokine profile following an additional stimulus of endotoxin in the serum of children with TBI compared to healthy controls at the time of injury. We repeated this at two weeks from injury, at timepoint when children with mTBI often appear to have recovered. Evoking and measuring an immune response in children who have sustained head injury may have a role in diagnostics, particularly in delineating recovery. This was explored using ratios of pro and anti-inflammatory markers.

\section{Methods \\ Study population}

This study was approved by the Ethics Committees of Children's Health Ireland (CHI) at Tallaght (Ref: 2016-03 (21); approved 28.03.16) and CHI at Temple Street Dublin (Ref: 16.019; approved 23.03.16), Ireland. All families and participants received verbal and documented information on the study. Written consent was obtained prior to recruitment. The following children were enrolled: a) mTBI: Emergency department attendees with Glasgow Coma Scale 14/15; b) sTBI: admitted to the Intensive Care with a Glasgow Coma Scale $\leq 8$ c) Paediatric Controls: children attending for phlebotomy or day case procedures with normal results and clinical outcomes. Children in all groups were excluded if they had recent fever or evidence of infection. Demographics that may have affect symptoms of concussion were collected included learning disability, prior medical history, visual disorders, motion sickness, migraine history, prior traumatic brain injury, family history of concussion, migraine or mental health disorders in first degree relatives (Additional file 1: Table S1). Mechanism of injury (Table 1), symptoms on presentation, at one hour and at the time of injury were recorded. A Child Sports Concussion Assessment Tool (Child-SCAT5), was recorded in children 5-12 years [22] and in children 13 years and older the SCAT5 was used [23] at the time of presentation. A post-concussive symptom inventory (PCSI) validated in children [24] was performed at the two-week assessment. Median time from injury at blood draw was four hours with an interquartile range of (1.75-22 h).

\section{Sample preparation}

Blood samples $(3 \mathrm{~mL})$ for in vitro experiments were collected in a sodium citrate anti-coagulated blood tube and processed within two hours of phlebotomy. Whole blood was incubated at $37^{\circ} \mathrm{C}$ for $1 \mathrm{~h}$ untreated (vehicle) or with Lipopolysaccharide (LPS; E.coli 0111:B4: SIGMA Life Science, Wicklow, Ireland) $10 \mathrm{ng} / \mathrm{ml}$. After incubation the samples were centrifuged at $10,000 \mathrm{rpm}$ for $10 \mathrm{~min}$ at room temperature. The plasma supernatant was stored at $-80^{\circ} \mathrm{C}$ for subsequent batch analysis [25].

\section{Cytokine analysis}

The following cytokines were evaluated using the MSD ${ }^{\circledR}$ MULTI-SPOT assay system from MesoScale Discovery, Rockville, MD, USA (www.meso-scale.com): IL-2, 
Table 1 Cytokines in children with TBI versus controls

\begin{tabular}{lccccc}
\hline Cytokine & Controls & Mild TBI & sTBI & p value* & p value ** \\
\hline IL-2 & $0.06(0.75-2.80)$ & $0.06(0.86-1.34)$ & $0.87(0.87-0.87)$ & 0.98 & 0.47 \\
IL-4 & $0.80(1.74-2.34)$ & $0.69(1.68-2.06)$ & $1.72(1.72-1.72)$ & 0.34 & $\mathbf{0 . 0 0 5}$ \\
IL-6 & $2.74(2.19-3.28)$ & $6.58(4.71-8.45)$ & $57.51(19.32-95.70)$ & $<0.0001$ \\
IL-8 & $70.69(61.19-80.18)$ & $26.9(21.02-32.97)$ & $102.2(3.70-200.8)$ & $<0.0001$ & 0.38 \\
IL-10 & $4.65(3.72-5.58)$ & $3.32(1.59-5.05)$ & $3.75(1.09-6.41)$ & $<0.0001$ & 0.72 \\
IL-17A & $14.98(8.02-21.93)$ & $1.48(1.14-1.82)$ & $1.39(0.78-1.99)$ & $<0.0001$ & 0.42 \\
TNF-a & $15.73(13.51-17.95)$ & $6.65(5.14-8.15)$ & $15.29(2.33-28.24)$ & $<\mathbf{0 0 0 1}$ & 0.82 \\
IFN-ץ & $107.9(75.63-140.1)$ & $203.2(-49.95-456.4)$ & $17.55(17.55-17.55)$ & $<\mathbf{0 . 0 0 0 1}$ & $\mathbf{0 . 0 1}$ \\
\hline
\end{tabular}

The mean, with upper and lower $95 \%$ Confidence Interval of the mean and $\mathrm{p}$-values of cytokine levels (pg/ml) in plasma of children with mild Traumatic brain injury $(n=110)$ and severe traumatic brain injury $(n=6)$ versus controls $(n=98)$. Cytokine values in bold denote $p<0.05$. ${ }^{*} p$ value: comparing controls to mTBI; ${ }^{* *} p$ value: comparing sTBI to $\mathrm{mTBI}$

IL-4, IL-6, IL-8, IL-10, IL-17A, IFN- $\gamma$ TNF- $\alpha$ [25]. Peripheral blood plasma was transferred to a 96 well MSD plate and these cytokines were assessed as per manufacturer's instructions. Assays were transferred to five U-PLEX platform plates with calibration curves showing expected signals, sensitivity precision, and accuracy. Sensitivities were $<1 \mathrm{pg} / \mathrm{ml}$ for many assays. All assays used the same diluents, diluting linearly from 1 -fourfold. Non-specific binding between assays was typically $<0.1 \%$. The assay reproducibility was calculated using the intra variation of the standard curves was shown to be within an acceptable range. The median of the lower limit of detection for all 5 plate runs were as follows: IL-2, IL-4, IL-6, IL-8, IL-10, IL-17A, IFN- $\gamma$, TNF- $\alpha, 0.87,1.72,0.84,0.38,0.62$, $1.15,17.55,2.39$ respectively, with a closely aligned average value, demonstrating the reproducibility of the assay. Where the sample was below the lower limit of detection the value of the lower limit of detection for that assay was substituted [26]. No samples reached an upper limit of detection.

\section{Statistics}

Descriptive statistics were used to describe the demographics of the cohort. The Kolmogorov-Smirnov test was used to check normality. Statistical analysis was performed using Mann Whitney tests to compare mean ranks between two independent cohorts. Significance was defined as $\mathrm{p}=0.05$. Results shown are expressed as mean \pm standard error of the mean (SEM) unless otherwise stated. Receiver operator curves were generated from baseline unstimulated serum cytokine levels. Data were analysed with GraphPad Prism v.7.

\section{Results}

\section{Clinical parameters}

There were 238 samples collected from 208 children enrolled in this study, including 110 children with TBI
(mTBI $\mathrm{n}=104$; sTBI $n=6)$ and 98 paediatric controls. In the mTBI group, 12 recruited patients declined phlebotomy and $30 \mathrm{mTBI}$ children had repeat serology at 2 weeks. The mean (SD) age of children enrolled was as follows: sTBI $(7.9 \pm 5.0) y$, male $(n=5)$, mild TBI $(11.2 \pm 4.0) y$ male $(n=69)$ and controls $(8.0 \pm 4.2) y$, male $(n=54)$. The sTBI cohort had a Glasgow Coma Scale (GCS) of less than 8 and required intubation, ventilation and an Intensive Care management. The causes of sTBI included falls from windows, car accidents, a crush injury and one fall. All had intracranial bleeding on CT imaging. Children in the mild TBI group had a GCS of 14/15 with the following causes: sports $(n=53 ; 51 \%)$, accidents including falls $(n=44 ; 42.3 \%)$ and road traffic accidents $(n=7 ; 6.7 \%)$. A loss of consciousness was described in $16.6 \%$ and $35.6 \%$ had amnesia. In the mild TBI group 26 (28.8\%) underwent CT imaging and 4 were abnormal: depressed skull fracture and intracranial bleed; small left frontotemporal contusion/contra-coup haemorrhagic lesion and non-displaced skull fractures $(n=2)$. In the mTBI cohort vomiting occurred in $41.8 \%$ with more than 3 episodes in $27.8 \%$. Full blood counts were available for 77 children at the same blood draw; median (interquartile range) white cell counts $8.4 \times 10^{9} / \mathrm{L}\left(6.5-11 \times 10^{9} / \mathrm{L}\right)$, neutrophils $4.5 \times 10^{9} / \mathrm{L}\left(3.3-8.3 \times 10^{9} / \mathrm{L}\right)$, lymphocytes $2.1 \times 10^{9} / \mathrm{L}\left(1.5-2.6 \times 10^{9} / \mathrm{L}.\right)$.

\section{Cytokines responses in Children with Traumatic brain injury versus controls at baseline}

Interleukin-6 was raised in all children with TBI compared to controls and was significantly higher in sTBI versus mTBI $(\mathrm{p}<0.0001$; Fig. 1$)$. All children with sTBI had IL-6 levels of $>20 \mathrm{pg} / \mathrm{ml}$ and no children with mTBI had values over $45 \mathrm{pg} / \mathrm{ml}$. This was highest in the first 0-12 h after injury (Additional file 2: Fig. S1) and was not significantly raised after this time point. At a cutoff value of $20 \mathrm{pg} / \mathrm{ml} \mathrm{IL-6}$ was 100\% sensitive and 99\% 


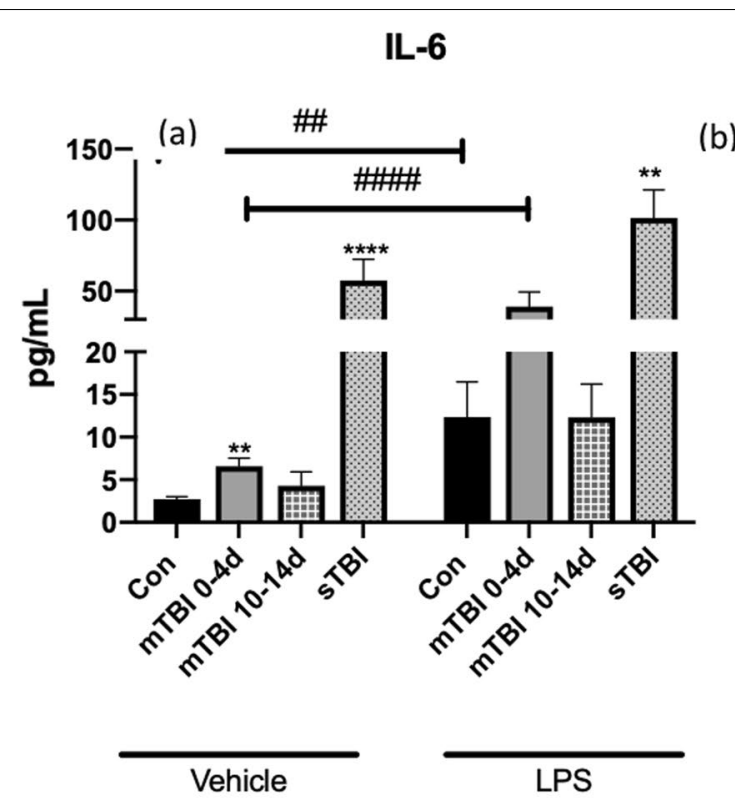

(c)

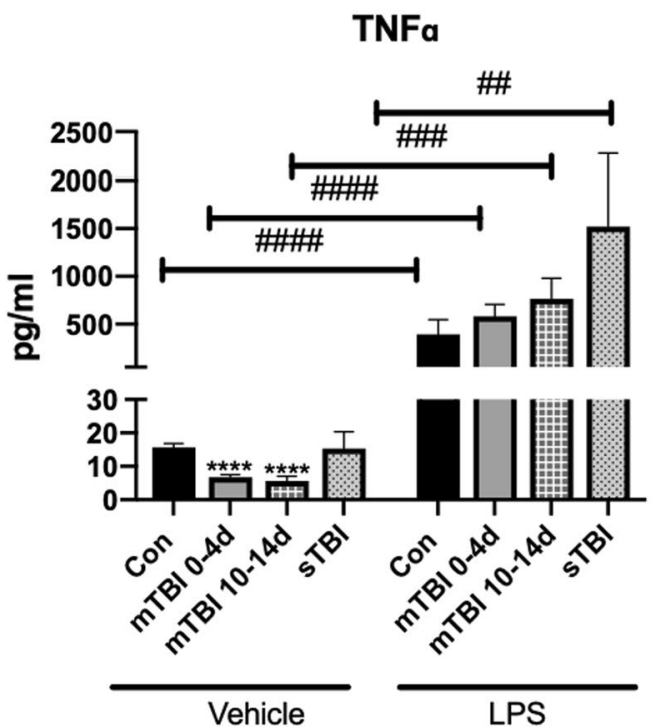

(b)

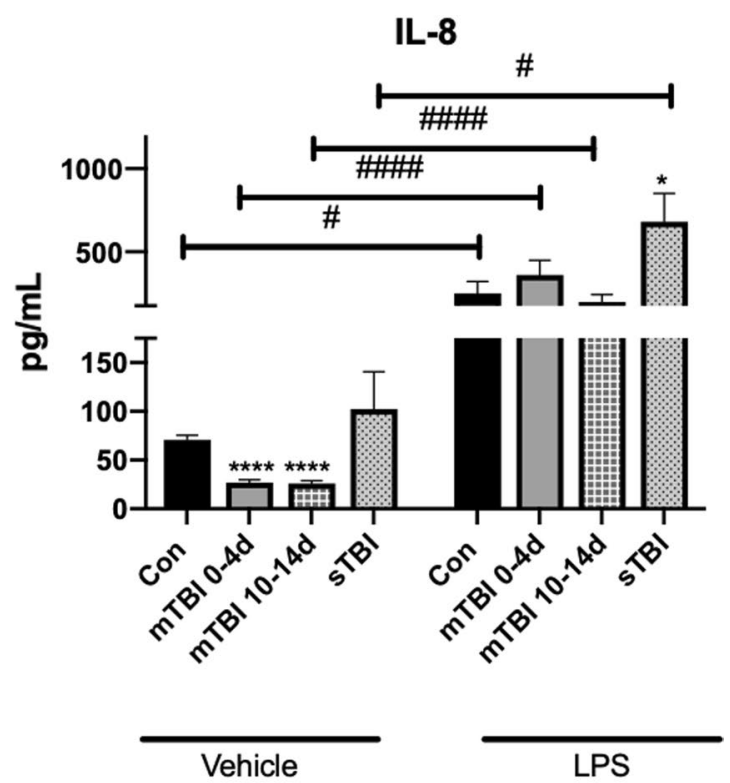

(d)

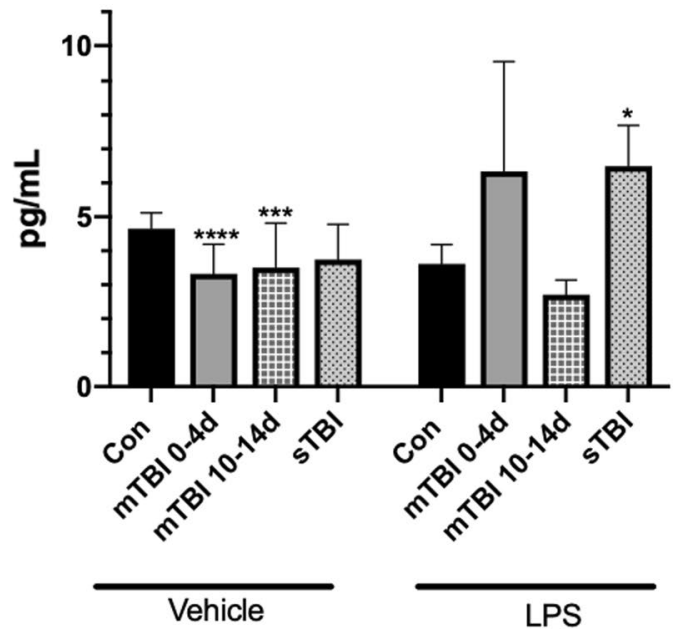

Fig. 1 Cytokines: a Interleukin (IL)-6, b IL-8, c TNFa and $\mathbf{d}$ IL-10 responses in Paediatric Traumatic brain injury. Serum of children with mild TBI (mTBI) at the time of presentation and at 2 weeks as well as children with severe TBI (STBI) at baseline and in response to LPS. Values expressed as pg/ $\mathrm{ml}$. Samples size (Vehicle: $n=104$ controls, $n=98 \mathrm{mTBI}$ at 0-4d, $n=29 \mathrm{mTBl}$ at 10-14d, $n=6$ severe TBI. LPS: $n=11$ controls, $n=18 \mathrm{mTBI}$ at 0-4d, $n=9$ mTBl at $10-14 d, n=4$ severe). ${ }^{*} p<0.05,{ }^{* *} p<0.01,{ }^{* * *} p<0.001,{ }^{* * * *} p<0.0001$, patient cohort compared to controls. \#=p<0.05 response to LPS from baseline. $\mathbf{a}{ }^{* *} p=0.005 \mathrm{mTBI} 0-4 \mathrm{~d}$ compared to controls, ${ }^{* * *} p<0.0001$ control compared to sTBI at baseline, \#\#p=0.001 controls pre and post LPS stimulation. \#\#\#\# $\mathrm{p}<0.0001 \mathrm{mTBI} 0-4 \mathrm{~d}$ pre and post LPS. ${ }^{* *} p=0.0015$ controls compared to sTBI within LPS group. $\mathbf{b}{ }^{* * *} p<0.0001$ mTBI $0=4 \mathrm{~d}$ compared to controls, ${ }^{* * * *} p<0.0001 \mathrm{mTBI}$ at $10-14 \mathrm{~d}$ compared to controls, ${ }^{*} p=0.035$ controls compared to sTBI in response to LPS. ${ }^{*} p=0.02$

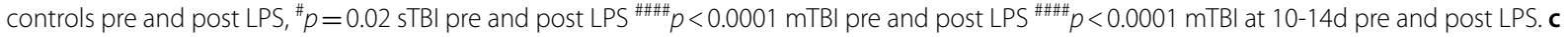
${ }^{* * * *} p<0.0001 \mathrm{mTBI} 0=4 \mathrm{~d}$ compared to controls, ${ }^{* * *} p<0.0001 \mathrm{mTBl}$ at $10-14 \mathrm{~d}$ compared to controls ${ }^{\# \#} p=0.009$ sTBI pre and post LPS \#\#\# $p=0.0001$ mTBI at 10-14d pre and post LPS ${ }^{\# \# \# \# ~} p<0.0001$ mTBI pre and post LPS, LPS \#\#\#\# $p<0.0001$ controls pre and post LPS. $\mathbf{d}^{* * * *} p<0.0001$ mTBI 0-4d compared to controls, ${ }^{* * *} p=0.0004 \mathrm{mTBI}$ at $10-14 \mathrm{~d}$ compared to controls, ${ }^{*} p=0.037$ controls compared to sTBI within LPS group 
(a)

IL-2

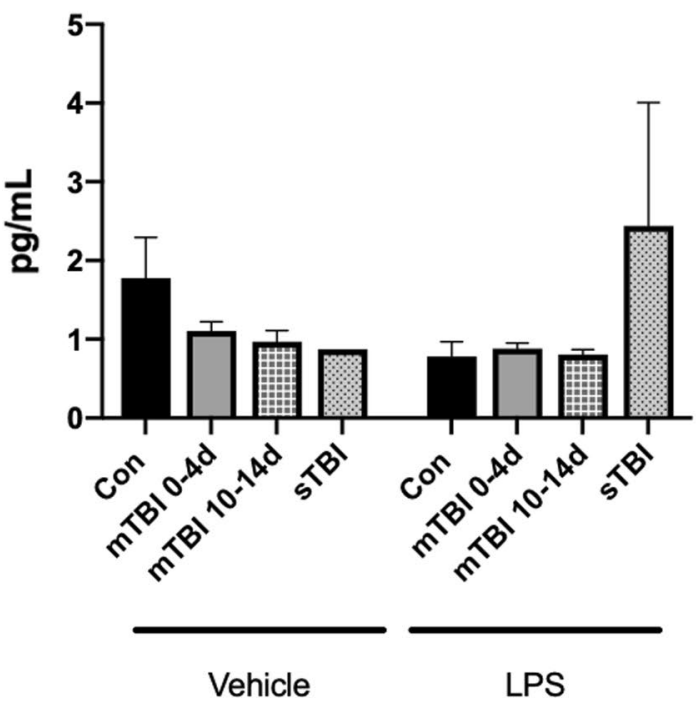

(c)

(b)

(d)

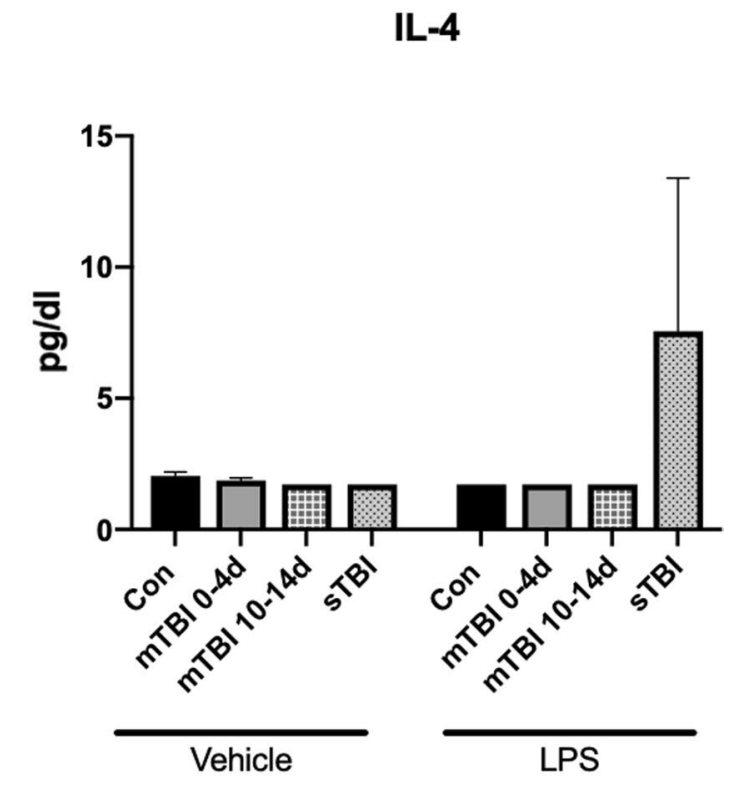

IFN-Y

IL17A
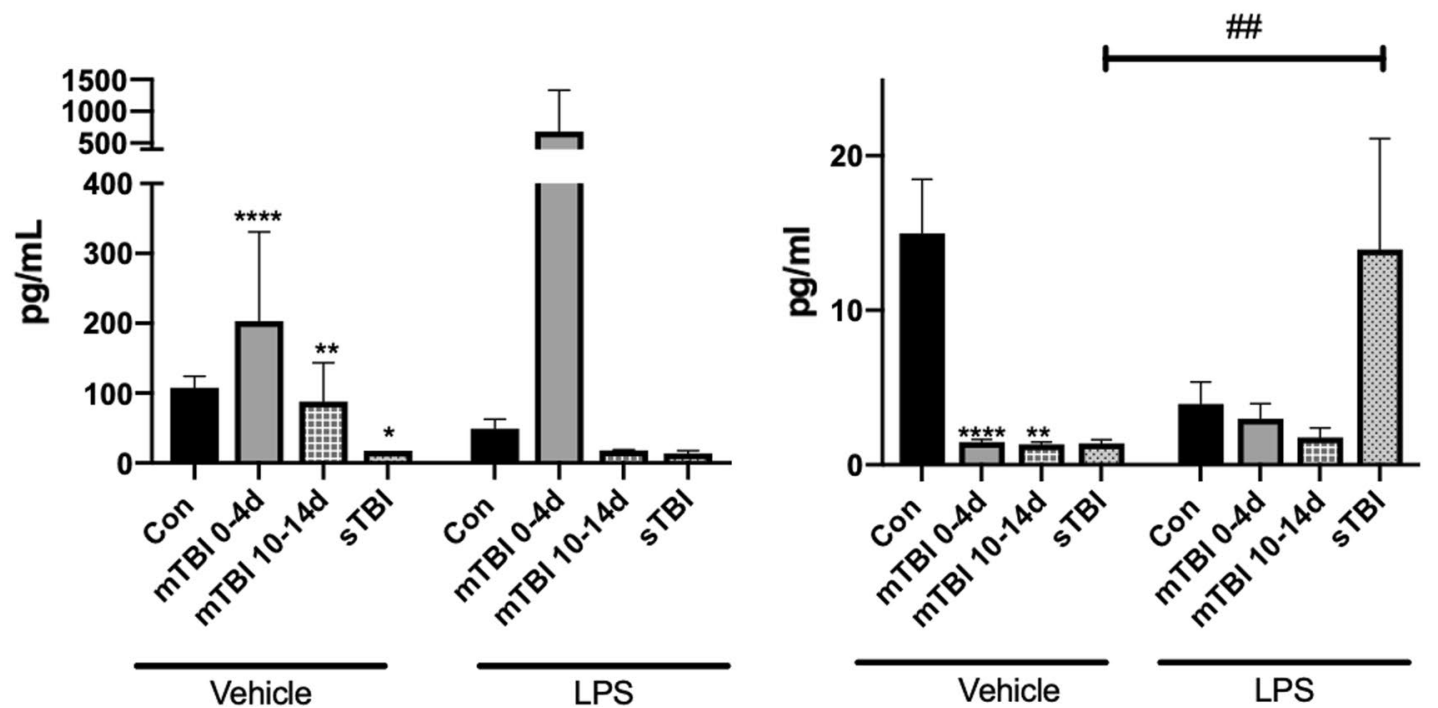

Fig. 2 Cytokines: a Interleukin 2 (IL)-2, b IL-4, c IFNy and $\mathbf{d} \mid \mathrm{L}-17 \mathrm{~A}$ responses in Paediatric Traumatic brain injury. Serum of children with mild TBI (mTBI) at the time of presentation and at 2 weeks as well as children with severe TBI (sTBI) at baseline and, in response to LPS. Values were expressed as pg/ml. Samples size (Vehicle: $n=104$ controls, $n=98 \mathrm{mTBl}$ at 0-4d, $n=29 \mathrm{mTBl}$ at 10-14d, $n=6$ severe TBI. LPS: $n=11$ controls, $n=18 \mathrm{mTBl}$ at $0-4 \mathrm{~d}, n=9 \mathrm{mTBl}$ at $10-14 \mathrm{~d}, n=4$ severe). ${ }^{*} p \leq 0.05^{*},{ }^{* *} p<0.01,{ }^{* * *} p<0.001,{ }^{* * * *} p<0.0001$, patient cohort compared to controls. \#=p<0.05 response to LPS from baseline. $\mathbf{a}, \mathbf{b}$ there was no statistical difference between groups. $\mathbf{c}^{* * * *} p<0.0001 \mathrm{mTBl}$ at baseline compared to controls, ${ }^{* *} p=0.0053 \mathrm{mTBl}$ at $10-14 \mathrm{~d}$ compared to controls, ${ }^{*} p=0.01$ severe compared to controls $\mathbf{d}^{* *} p=0.0097$ control compared to mTBI at $10-14 \mathrm{~d}$, ${ }^{* * * *} p<0.0001$ control compared to mTBI at $0-4 d,{ }^{\# \#} p=0.0048$ sTBI pre and post LPS stimulation 
Table 2 Receiver operator characteristic curves for cytokines of children with mild Traumatic brain injury and controls

\begin{tabular}{lll}
\hline Cytokine & $\begin{array}{l}\text { mTBI at 0-4d } \\
\text { AUC }\end{array}$ & $\begin{array}{l}\text { mTBI at 10-14d } \\
\text { AUC }\end{array}$ \\
\hline $\begin{array}{ll}\text { Prediction of mTBI } \\
\text { IL-6 }\end{array}$ & \\
TNF-a & 0.61 & 0.464 \\
IL6/IL8 & 0.204 & 0.163 \\
IL6/L10 & 0.829 & 0.744 \\
IL6/L-17A & 0.801 & 0.686 \\
IL6/IL10/IL-17A & 0.744 & 0.621 \\
\hline
\end{tabular}

specific for sTBI and 91\% sensitive for mTBI detection but non-specific. Interferon- $\gamma$ was decreased in sTBI $(\mathrm{p}=0.01)$ but raised in mTBI $(\mathrm{p}<0.0001$; Table 1 , Fig. 2$)$. Although in children with sTBI IL-8, IL-10 IL-17A and TNF- $\alpha$, did not change these cytokines were decreased in children with mTBI versus controls (Table 2 \& Figs. 1, 2 ). There were no significant differences seen in IL-2 or IL-4 in mild or sTBI compared to controls (Table 2 \& Fig. 2). While patients with mTBI had significant but less potent increases in IL-6 concentrations than sTBI, they had a rise in IFN- $\gamma$, and reduction in IL-8, IL-10, IL-17A and TNF- $\alpha$ concentrations. There was no significant difference between gender (Additional file 2: Fig. S2). There were higher levels of IL- 8 and TNF- $\alpha$ in younger boys (Additional file 2: Fig. S3) while IL-10 was lower in older girls (Additional file 2: Fig. S4).

\section{Cytokines to identify traumatic brain injury in children at presentation}

To further examine the utility of cytokine measurement and ratios of pro- and anti-inflammatory markers in the setting of TBI, receiver operating characteristic curves were used to select patients with TBI compared to controls (Table 2, Additional file 2: Fig. S5). Severe TBI were distinguished from control patients by IL-6 with $100 \%$ sensitivity and specificity, with an Area Under the Curve (AUC) of 1 . Mild TBI is less obvious clinically and biochemically and the presence of mild TBI was indicated by IL- 6 with an AUC of 0.61 CI (0.54-0.691). The ratio of IL-6/IL- 8 gave the highest AUC of 0.829 to select mild TBI. Since IL-10 and IL-17A were decreased in both mild and severe TBI compared to controls we examined the utility of using these to create a ratio which would be useful across the continuum from mild to severe. Using IL-6/IL-10 increased the AUC to predict mTBI to 0.801 CI (0.738-0864). Using IL-6/IL-10/IL-17A the AUC was 0.832 at $0-4 d$.

\section{Effects of ex-vivo LPS stimulation on Cytokine responses} in children with TBI and controls

To challenge the immune system in the setting of TBI whole blood was stimulated with LPS. In sTBI, LPS stimulation caused a significant rise in IL- 8, TNF- $\alpha$ and IL-17A from baseline $(p<0.000001, p<0.000001$, $p=0.0048)$. Baseline levels of IL-6 in the sTBI cohort were high compared to both mTBI and controls and were not significantly increased with LPS (Fig. 1). Prior to stimulation with LPS, IL-8 and IL-10 levels were not significantly higher in sTBI compared to controls $(p=0.38$, $p=0.7)$. Following LPS stimulation, while all groups had increased cytokine production there was a significant increase in IL-8 and IL-10 levels in sTBI compared to stimulated control samples $(p=0.035, p=0.037)$. In paediatric controls and in children with mTBI, LPS stimulation significantly increased IL-8, TNF- $\alpha$ and also IL-6 levels $(<0.0001,<0.0001,<0.0001$; Fig. 1$)$. In children with mTBI baseline IL- 8 and TNF- $\alpha$ levels were low but were significantly responsive to LPS beyond the response seen for controls (Table 2). A significant response to LPS stimulation for IL-8 and TNF- $\alpha(p<0.0001, p=0.0002$; Table 2) was seen in the serum taken from children with mTBI at 10-14 days from injury. Interleukin-2 and IL-4 did not respond to LPS stimulation in any group. Stimulation with LPS unmasked a hyperresponsiveness of the immune system in children who had both sTBI and mTBI. Children with mTBI specifically had lower levels of cytokines until stimulated with LPS.

\section{Persistent inflammatory response}

Due to the persistence of the immune response for an undefined period of time after TBI, we followed the immune response in these patients for two weeks. In children with mTBI at 2 weeks, IL-17A, IL-8, IL-10 and TNF- $\alpha$ levels remained significantly decreased $(p=0.009$, $p<0.0001, p=0.0006, p<0.0001$, respectively), while IL-6 returned to baseline (Figs. 1 and 2). In mTBI, IL-8 responsiveness to LPS peaked at 0-4 days but remained above control levels at 10-14 days (Additional file 3: Table S2). In mTBI LPS induced a threefold increase in IFN- $\gamma$ levels on 0-4 day. This phenomen was not seen at 2 weeks where LPS induced a decreased response. There was a greater TNF- $\alpha$ production in response to LPS in mTBI at 10-14 days than at 0-4 day and versus controls (Table 2). At 10-14 days from injury the serum of children with mild TBI showed persistent cytokine levels with an ROC curve using a ratio of IL-6/IL-10/IL-17A to give an AUC of 0.746. The cytokine production seen in children with mTBI persisted for 2 weeks as well as the response to LPS. 


\section{Discussion}

At the time of injury, in children with both sTBI and mTBI, IL-6 was significantly elevated compared to controls. When stimulated ex-vivo with LPS, in sTBI and not mTBI, significant elevations of IL-8 and IL-10 were seen compared to controls. It is well-known that there is an alteration in the immune profile following sTBI in adults and children [12]. The injury profile in mTBI is not on a continuum with sTBI, mTBI having no gross injuries on imaging. In recent studies, blast injury has too, been shown to be distinct [27]. An adult mouse model has shown increased remote lung neutrophil migration in the cohort with mTBI compared to controls [28]. No pediatric study has shown sustained systemic immune change following mTBI. This mTBI cohort showed significantly lower IL-8, IL-10 IL-17A and TNF- $\alpha$ at the time of injury. However, when stimulated with LPS, these children with mTBI had greater IL- 8 and TNF- $\alpha$ responses, both in serum from the time of injury and serum at 2 weeks, showing persistent immune activation, at a time when the majority of children show clinical recovery. Sex hormones have a role to play in TBI, with the oestrogen in female puberty being protective [29]. While there were no significant differences in gender, older boys had lower IL-8 and TNF- $\alpha$ responses, while older females had lower IL- 10 . Due to the smaller number of female patients in the study, and due to the heterogeneous nature of injuries in the cohort this should be interpreted with caution. Serum taken 2 weeks from injury showed the same pattern of significantly decreased IL-17A, IL-8, IL-10 and TNF- $\alpha$ levels, while IL- 6 had returned to baseline. The systemic immune response to mTBI in children is a reduction of cytokine production by myeloid cells. While this effect persists, if provoked by endotoxin, the response of the peripheral myeloid cells in children with $\mathrm{mTBI}$ is exaggerated.

There are gaps in our understanding in how to modify the innate immune response to trauma to better the outcomes in TBI across the spectrum, particularly in children where the innate immune responses are different. Limiting the pro-inflammatory response and promoting anti-inflammatory properties is key to therapeutics. Defining these responses and timelines are important for secondary injury, particularly in the milder TBI children, not previously known to have persistent immune dysregulation. Early measurement of innate immune function may better help to risk stratify in mTBI.

Interleukin- 6 was elevated in all children with TBI and predicted sTBI. IL-6/IL-10 ratio enhanced the prediction of mTBI relative to IL- 6 alone. In sports related concussion IL-6 levels correlated with symptom burden in young adults [11]. While IL-6 is elevated in MTBI in adult studies, it has not correlated with cognitive impairment [30], a common component of post concussive syndrome. Gill and colleagues showed significant increases in IL-6, IL-10 and TNF- $\alpha$ during blast exposure in military training [31]. In one study IL- 6 and TNF- $\alpha$ were raised in mTBI, but TNF- $\alpha$ was only raised in those with evidence of injury seen on CT, and not in those with injury only evident on MRI [32]. In our study IL-6 elevations did not persist at 2 weeks. Interestingly exercise prior to injury in animal models have been shown to attenuate levels of IL-6 in response to mTBI [33], and also the effect of the injury [34].

In childhood, sTBI is associated with increased serum IL-8 and predicted a worse outcome [10]. In neonatal brain injury elevated IL- 8 and IL-10 correlated with poor outcome and severity of injury [35]. Most TBI studies of cytokine responses have been in sTBI but not mTBI, where there is no overt intracranial bleeding, and the mechanism of injury is different. We demonstrated an opposite effect in this cohort with significantly lower levels of IL-8 and IL-10 in our pediatric mTBI cohort at baseline when compared to controls. An adult study looking at blast-related TBI in the military found similar responses to our study: IL-8 decreased from pre-deployment levels in those who suffered blast injury [27]. Interestingly this effect was more profound in those younger than 25 years. This may be an effect of the injury type or of age or may also suggest the response to all brain injury is not on a continuum.

There was a significant and sustained decrease in IL17A following TBI. Only in sTBI did LPS mediate an IL-17A response to stimulation. Interleukin-17A is associated with TBI severity and also progression in multiple sclerosis. It has a role in mediating the blood-brain barrier [36]. A sTBI model in rats showed increased IL-17 production [37]. The role of the adaptive immune system in TBI, particularly in repeated mTBI needs further clarification.

Two weeks from injury there was a sustained decrease in IL-8, IL-10 IL-17A and TNF- $\alpha$ in the mTBI cohort. Animal studies demonstrate persistent immune dysfunction in sTBI [6] and chronic changes associated with repeated head injury [38]. The mouse model of head injury showed chronic persistent changes in thymic and T-cell function, neutrophil and monocyte function, and proinflammatory cytokine production including TNF- $\alpha$, at 60 days [6]. In moderate/severe blast injury there was a reduction in IL-10, IL-17A and TNF- $\alpha$ even at a year from injury reflecting persistent inflammation [39]. Following neonatal encephalopathy children have persistent immune dysfunction much longer, at school-age [7]. Jeschke and colleagues demonstrated altered immune response in the three years following a severe physical 
burn injury in children [40]. The response of the immune system over time to one TBI and repeated insults may be amenable to immunomodulation.

Immunomodulatory effects of TBI are demonstrated in both cohorts. Provocative testing with LPS stimulation of samples from children with TBI unmasks immune dysregulation and may aid diagnostics. sTBI had significantly elevated IL-8 and IL-10 response to LPS while mTBI had a significant and sustained IL- 6 and IL-8 response to LPS challenge, at time of injury and at two weeks. The greater TNF- $\alpha$ response at 10-14 days was specific to $\mathrm{mTBI}$, suggesting priming of peripherally circulating immune cells. Prior studies of TNF- $\alpha$ responsive to LPS looked only at sTBI and not mTBI. A blunted TNF- $\alpha$ response in STBI to LPS stimulation has been previously demonstrated in sepsis and trauma in children [41, 42]. This phenomenon results in increased nosocomial infections [43]. Our study is the first to look at children with $\mathrm{mTBI} /$ concussion which shows priming of the TNF- $\alpha$ response from myeloid cells to LPS at two weeks, with an opposite and greater response to LPS challenge, at time when the majority of children appear to have recovered.

Innate immune function in children is distinctly different to adults. Ageing is associated with low grade chronic systemic inflammation with elevated IL-6 and C-reactive protein [44]. Studying the effects of TBI in children naïve to chronic disease offers a unique opportunity to look at TBI in isolation. Understanding the pathophysiological and post inflammatory changes associated with paediatric TBI may facilitate the understanding of potential new drug therapies in the initial and chronic phases of TBI. Even the use and timing of basic anti-inflammatory medication is poorly understood. This study was limited by the small number of sTBI, but the large number in the mTBI cohort was a strength in this under studied population.

\section{Conclusion}

This study demonstrated immune dysfunction not only in sTBI but also in mTBI. It established that immune dysfunction is persistent following paediatric mTBI. Interleukin- 6 was elevated across the spectrum of TBI. The effect of TBI on systemic inflammation warrants serial follow up for months and years. Evidence of injury in mTBI is occult, usually described subjectively by symptom report. A strong biomarker of mTBI has alluded scientific research, however provocative immune testing may be most beneficial. Further validation of these cytokines is key to understanding the mechanisms that are also a target of immunomodulatory therapy.

\section{Abbreviations}

CT: Computerised tomography; GCS: Glasgow coma scale; IFN-ү: Interferon- $\gamma$; IL: Interleukin; LPS: Lipopolysaccharide; mTBI: Mild traumatic brain injury; PCSI: Post concussive symptom inventory; SCAT: Sports concussion assessment tool; sTBI: Severe traumatic brain injury; TBI: Traumatic brain injury; TNF-a: Tumour necrosis factor-a.

\section{Supplementary Information}

The online version contains supplementary material available at https://doi. org/10.1186/s12974-022-02390-5.

Additional file 1. Table S1. Detailed demographics of children with TBI. Additional file 2. Figure S1. Cytokines in Paediatric Traumatic brain injury at time epochs from injury. Figure S2. Cytokines in Paediatric Traumatic brain injury by gender. Figure S3. Cytokines in Male Paediatric Traumatic brain injury by age group. Figure S4. Cytokines in Female Paediatric Traumatic brain injury by age group. Figure S5. Receiver operator characteristic curves for predicting presence $\mathbf{a}, \mathbf{b}$ and absence $\mathbf{c}$, $\mathbf{d}$ of $\mathrm{mTBI}$ compared to controls.

Additional file 3. Table S2. LPS induced cytokine responses in children with $\mathrm{mTBI}$ versus controls.

\section{Acknowledgements \\ The patients and parents who took part in this study.}

\section{Author's contributions}

The manuscript is being submitted on behalf of all the authors and is the original work of all authors. ER was responsible for recruitment, sample acquistion, lab experiments, analysis and was responsible for writing the main draft of the manuscript. LK and DH added expert laboratory assisstance. LK, $\mathrm{DH}, \mathrm{DMC}, \mathrm{CS}, \mathrm{ED}, \mathrm{AL}, \mathrm{GB}$ were responsible for patient recruitment, sample acquisition, reviewing and editing the manuscript. LKTB and EM were key in study design, supervising the research and its outcomes and providing crucial editorial assistance. All authors had editorial license to review and re-draft the manuscript, and all contributors had to approve the final edit. All authors are accountable for the accuracy and scientific integrity of this work. All authors read and approved the final manuscript.

\section{Funding}

This study was funded by the National Children's Hospital Fund [206965], Tallaght, Dublin, Ireland.

\section{Availability of data and materials}

The experimental data used to support the findings of this study are available from the corresponding author upon request.

\section{Declarations}

\section{Ethics approval and consent to participate}

This study was approved by the Ethics Committees of Children's Health Ireland (CHI) at Tallaght (Ref: 2016-03 (21); approved 28.03.16) and CHI at Temple Street Dublin (Ref: 16.019; approved 23.03.16), Ireland. Informed consent was obtained from patients and/or parents.

\section{Consent for publication}

Not applicable.

\section{Competing interests}

The authors have no conflict of interest to declare.

\section{Author details}

${ }^{1}$ Department of Paediatrics, Trinity College, The University of Dublin, Dublin, Ireland. ${ }^{2}$ Trinity Translational Medicine Institute (TTMI), Trinity Centre for Health Sciences, Tallaght University Hospital, Dublin 24, Ireland. ${ }^{3}$ Department of Medicine, Trinity Centre for Health Sciences, Trinity Research in Childhood Centre (TRiCC), Trinity Translational Medicine Institute, St James Hospital, Dublin 8, 
Ireland. ${ }^{4}$ Paediatric Emergency Medicine, Children's Hospital Ireland (CHI) at Tallaght, Dublin 24, Ireland. ${ }^{5}$ Department of Biochemistry, Tallaght University Hospital, Dublin 24, Ireland. ${ }^{6} \mathrm{Critical}$ Care Medicine, Children's Health Ireland (CHI) at Temple Street, Dublin 1, Ireland. ${ }^{7}$ Department of Neonatology, $\mathrm{CHI}$ at Crumlin, Dublin, Ireland. ${ }^{8}$ Department of Neonatology, Coombe Women and Infants University Hospital Dublin, Dublin, Ireland. 'Department of Paediatric Emergency Medicine, Royal Children's Hospital, Melbourne, Australia.

${ }^{10}$ Department of Paediatrics, Waterford Regional Hospital, Waterford, Ireland.

Received: 31 May 2021 Accepted: 14 January 2022

Published online: 07 February 2022

\section{References}

1. Ryan E, Bolger T, Barrett MJ, Blackburn C, Okafor I, McNamara R, Molloy EJ. Paediatric head injury and traumatic brain injury. Ir Med J. 2020;113:94.

2. Koepsell TD, Rivara FP, Vavilala MS, Wang J, Temkin N, Jaffe KM, Durbin DR. Incidence and descriptive epidemiologic features of traumatic brain injury in King County, Washington. Pediatrics. 2011;128:946-54.

3. Nasr IW, Chun Y, Kannan S. Neuroimmune responses in the developing brain following traumatic brain injury. Exp Neurol. 2019:320:112957.

4. Monti D, Ostan R, Borelli V, Castellani G, Franceschi C. Inflammaging and human longevity in the omics era. Mech Ageing Dev. 2017:165:129-38.

5. Aldrimer M, Ridefelt P, Rödöö P, Niklasson F, Gustafsson J, Hellberg D. Population-based pediatric reference intervals for hematology, iron and transferrin. Scand J Clin Lab Invest. 2013;73:253-61.

6. Ritzel RM, Doran SJ, Barrett JP, Henry RJ, Ma EL, Faden AI, Loane DJ. Chronic Alterations in Systemic Immune Function after Traumatic Brain Injury. J Neurotrauma. 2018;35:1419-36.

7. Zareen Z, Strickland T, Eneaney VM, Kelly LA, McDonald D, Sweetman D, Molloy EJ. Cytokine dysregulation persists in childhood post Neonatal Encephalopathy. BMC Neurol. 2020;20:115.

8. Frugier T, Morganti-Kossmann MC, O'Reilly D, McLean CA. In situ detection of inflammatory mediators in post mortem human brain tissue after traumatic injury. J Neurotrauma. 2010;27:497-507.

9. Bell MJ, Kochanek PM, Doughty LA, Carcillo JA, Adelson PD, Clark RS, Whalen MJ, DeKosky ST. Comparison of the interleukin-6 and interleukin-10 response in children after severe traumatic brain injury or septic shock. Acta Neurochir Suppl. 1997;70:96-7.

10. Lo TY, Jones PA, Minns RA. Combining coma score and serum biomarker levels to predict unfavorable outcome following childhood brain trauma. J Neurotrauma. 2010;27:2139-45.

11. Nitta ME, Savitz J, Nelson LD, Teague TK, Hoelzle JB, McCrea MA, Meier TB. Acute elevation of serum inflammatory markers predicts symptom recovery after concussion. Neurology. 2019:93:e497-507.

12. Sribnick EA, Hensley J, Moore-Clingenpeel M, Muszynski JA, Thakkar RK, Hall MW. Nosocomial infection following severe traumatic injury in children. Pediatr Crit Care Med. 2020;21:443-50.

13. Rodney T, Osier N, Gill J. Pro- and anti-inflammatory biomarkers and traumatic brain injury outcomes: A review. Cytokine. 2018;1 10:248-56.

14. Grilli M, Barbieri I, Basudev H, Brusa R, Casati C, Lozza G, Ongini E. Interleukin-10 modulates neuronal threshold of vulnerability to ischaemic damage. Eur J Neurosci. 2000;12:2265-72.

15. Soares FM, de Souza NM, Schwarzbold ML, Diaz AP, Nunes JC, Hohl A, da Silva PN, Vieira J, de Souza RL, Bertotti MM, et al. Interleukin-10 is an independent biomarker of severe traumatic brain injury prognosis. NeurolmmunoModulation. 2012;19:377-85.

16. Yang GY, Gong C, Qin Z, Liu XH, Lorris Betz A. Tumor necrosis factor alpha expression produces increased blood-brain barrier permeability following temporary focal cerebral ischemia in mice. Brain Res Mol Brain Res. 1999;69:135-43.

17. Shohami E, Bass R, Wallach D, Yamin A, Gallily R. Inhibition of tumor necrosis factor alpha (TNFa) activity in rat brain is associated with cerebroprotection after closed head injury. J Cereb Blood Flow Metab. 1996;16:378-84.

18. Hasturk AE, Gokce EC, Yilmaz ER, Horasanli B, Evirgen O, Hayirli N, Gokturk $H$, Erguder I, Can B. Therapeutic evaluation of tumor necrosis factor-alpha antagonist etanercept against traumatic brain injury in rats: ultrastructural, pathological, and biochemical analyses. Asian J Neurosurg. 2018;13:1018-25.
19. Li HL, Kostulas N, Huang YM, Xiao BG, van der Meide P, Kostulas V, Giedraitas V, Link H. IL-17 and IFN-gamma mRNA expression is increased in the brain and systemically after permanent middle cerebral artery occlusion in the rat. J Neuroimmunol. 2001;116:5-14.

20. McGinley AM, Sutton CE, Edwards SC, Leane CM, DeCourcey J, Teijeiro A, Hamilton JA, Boon L, Djouder N, Mills KHG. Interleukin-17A serves a priming role in autoimmunity by recruiting IL-1 beta-producing myeloid cells that promote pathogenic T cells. Immunity. 2020;52:342-356.e346.

21. Berer K, Mues M, Koutrolos M, Rasbi ZA, Boziki M, Johner C, Wekerle H, Krishnamoorthy G. Commensal microbiota and myelin autoantigen cooperate to trigger autoimmune demyelination. Nature. 2011;479:538-41.

22. Davis GA, Purcell L, Schneider KJ, Yeates KO, Gioia GA, Anderson V, Ellenbogen RG, Echemendia RJ, Makdissi M, Sills A, et al. The child sport concussion assessment tool 5th edition (Child SCAT5): background and rationale. Br J Sports Med. 2017;51:859-61.

23. Echemendia RJ, Broglio SP, Davis GA, Guskiewicz KM, Hayden KA, Leddy $\mathrm{JJ}$, Meehan WP, Putukian M, Sullivan SJ, Schneider KJ, McCrory P. What tests and measures should be added to the SCAT3 and related tests to improve their reliability, sensitivity and/or specificity in sideline concussion diagnosis? A systematic review. Br J Sports Med. 2017;51:895.

24. Sady MD, Vaughan CG, Gioia GA. Psychometric characteristics of the postconcussion symptom inventory in children and adolescents. Arch Clin Neuropsychol. 2014;29:348-63.

25. Huggard D, Kelly L, Ryan E, McGrane F, Lagan N, Roche E, Balfe J, Leahy TR, Franklin O, Doherty DG, Molloy EJ. Increased systemic inflammation in children with Down syndrome. Cytokine. 2020;127:154938.

26. Shoari N, Dube JS. Toward improved analysis of concentration data: embracing nondetects. Environ Toxicol Chem. 2018;37:643-56.

27. Rusiecki J, Levin LI, Wang L, Byrne C, Krishnamurthy J, Chen L, Galdzicki Z, French LM. Blast traumatic brain injury and serum inflammatory cytokines: a repeated measures case-control study among US military service members. J Neuroinflam. 2020;17:20.

28. Humphries DC, O'Neill S, Scholefield E, Dorward DA, Mackinnon AC, Rossi AG, Haslett C, Andrews PJD, Rhodes J, Dhaliwal K. Cerebral concussion primes the lungs for subsequent neutrophil-mediated injury. Crit Care Med. 2018;46:e937-44.

29. Shahrokhi N, Khaksari M, Soltani Z, Mahmoodi M, Nakhaee N. Effect of sex steroid hormones on brain edema, intracranial pressure, and neurologic outcomes after traumatic brain injury. Can J Physiol Pharmacol. 2010;88:414-21.

30. Sun Y, Bai L, Niu X, Wang Z, Yin B, Bai G, Zhang D, Gan S, Sun C, Wang S, et al. Elevated serum levels of inflammation-related cytokines in mild traumatic brain injury are associated with cognitive performance. Front Neurol. 2019;10:1120.

31. Gill J, Motamedi V, Osier N, Dell K, Arcurio L, Carr W, Walker P, Ahlers S, Lopresti M, Yarnell A. Moderate blast exposure results in increased IL-6 and TNFalpha in peripheral blood. Brain Behav Immun. 2017;65:90-4.

32. Edwards KA, Pattinson CL, Guedes VA, Peyer J, Moore C, Davis T, Devoto C, Turtzo LC, Latour L, Gill JM. Inflammatory cytokines associate with neuroimaging after acute mild traumatic brain injury. Front Neurol. 2020;11:348.

33. Febbraio MA, Pedersen BK. Contraction-induced myokine production and release: is skeletal muscle an endocrine organ? Exerc Sport Sci Rev. 2005;33:114-9.

34. Anderson V, Manikas V, Babl FE, Hearps S, Dooley J. Impact of moderate exercise on post-concussive symptoms and cognitive function after concussion in children and adolescents compared to healthy controls. Int J Sports Med. 2018;39:696-703.

35. O'Hare FM, Watson RW, O'Neill A, Segurado R, Sweetman D, Downey P, Mooney E, Murphy J, Donoghue V, Molloy EJ. Serial cytokine alterations and abnormal neuroimaging in newborn infants with encephalopathy. Acta Paediatr. 2017;106:561-7.

36. Caccamo N, La Mendola C, Orlando V, Meraviglia S, Todaro M, Stassi G, Sireci G, Fournie JJ, Dieli F. Differentiation, phenotype, and function of interleukin-17-producing human Vgamma9Vdelta2 T cells. Blood. 2011;118:129-38.

37. Li T, Zhang YM, Han D, Hua R, Guo BN, Hu SQ, Yan XL, Xu T. Involvement of IL-17 in secondary brain injury after a traumatic brain injury in rats. NeuroMol Med. 2017;19:541-54. 
38. Loane DJ, Kumar A, Stoica BA, Cabatbat R, Faden Al. Progressive neurodegeneration after experimental brain trauma: association with chronic microglial activation. J Neuropathol Exp Neurol. 2014;73:14-29.

39. Chen Y, Huang W, Constantini S. The differences between blast-induced and sports-related brain injuries. Front Neurol. 2013:4:119.

40. Jeschke MG, Gauglitz GG, Kulp GA, Finnerty CC, Williams FN, Kraft R, Suman OE, Mlcak RP, Herndon DN. Long-term persistance of the pathophysiologic response to severe burn injury. PLOS ONE. 2011;6:e21245.

41. Muszynski JA, Nofziger R, Moore-Clingenpeel M, Greathouse K, Anglim L, Steele L, Hensley J, Hanson-Huber L, Nateri J, Ramilo O, Hall MW. Early Immune Function and Duration of Organ Dysfunction in Critically III Children with Sepsis. Am J Respir Crit Care Med. 2018;198:361-9.

42. Muszynski JA, Nofziger R, Greathouse K, Nateri J, Hanson-Huber L, Steele L, Nicol K, Groner Jl, Besner GE, Raffel C, et al. Innate immune function predicts the development of nosocomial infection in critically injured children. Shock. 2014;42:313-21.

43. Holland MC, Mackersie RC, Morabito D, Campbell AR, Kivett VA, Patel R, Erickson VR, Pittet JF. The development of acute lung injury is associated with worse neurologic outcome in patients with severe traumatic brain injury. J Trauma. 2003;55:106-11.

44. Marcos-Pérez D, Sánchez-Flores M, Proietti S, Bonassi S, Costa S, Teixeira JP, Fernández-Tajes J, Pásaro E, Laffon B, Valdiglesias V. Association of inflammatory mediators with frailty status in older adults: results from a systematic review and meta-analysis. Geroscience. 2020. https://doi.org/ 10.3390/nu12082286.

\section{Publisher's Note}

Springer Nature remains neutral with regard to jurisdictional claims in published maps and institutional affiliations.

- fast, convenient online submission

- thorough peer review by experienced researchers in your field

- rapid publication on acceptance

- support for research data, including large and complex data types

- gold Open Access which fosters wider collaboration and increased citations

- maximum visibility for your research: over 100M website views per year

At BMC, research is always in progress.

Learn more biomedcentral.com/submissions 